\title{
¿Por qué delinquen nuestros niños? Niñez y publicaciones criminológicas (Buenos Aires, 1930-1946)
}

DOI: 10.15175/1984-2503-202214105

Mariana Dovio*

\section{Resumen}

Hacia 1930 en Buenos Aires, médicos y abogados se preguntaron por qué delinquían los niños, como parte de una agenda penal que se visualizó en publicaciones como Revista de Criminología, Psiquiatría y Medicina Legal y Anales de la Sociedad Argentina de Criminología entre 1930 y 1946. Desde sus discursos, concebidos como una práctica social, se abordó la peligrosidad en la niñez, vinculada a cualidades biológicas, aspectos ambientales, familiares y de género que eran señalados como escenario previo al delito. Se retomaron avances de la endocrinología, biotipología criminal y psiquiatría. Estos temas también fueron abordados en el Primer Congreso Internacional de Criminología en Roma y el Primer Congreso Latinoamericano de Criminología en Buenos Aires, ambos celebrados en 1938. A partir de un análisis cualitativo del discurso habría habido una mixtura en la incidencia de lo biológico y lo social en la peligrosidad, en la niñez.

Palabras claves: niñez; maternidad; delincuencia; pobreza.

\section{Por que os nossos filhos se tornam delinquentes? Infância e publicações criminológicas (Buenos Aires, 1930-1946)}

\section{Resumo}

Em Buenos Aires na década de 1930, no âmbito de pesquisas criminológicas encontradas em publicações como a Revista de Psiquiatría, Criminología y Medicina Legal e nos Anales da Sociedad Argentina de Criminología, entre 1930 e 1946, médicos e advogados se perguntavam por que as crianças podiam se tornar delinquentes. Nestes discursos, concebidos como uma prática social, a periculosidade da infância foi abordada em conexão com as especificidades biológicas e os aspectos ambientais, familiares e de gênero descritos como fatores propícios ao crime. Contava-se então com os avanços em endocrinologia, biotipologia criminal e psiquiatria. Estes temas foram igualmente abordados por ocasião do Primeiro Congresso Internacional de Criminologia, em Roma, e do Primeiro Congresso Latino-Americano de Criminologia, em Buenos Aires, ambos em 1938. A partir de uma análise qualitativa do discurso, identificamos à época a defesa de uma mistura de incidência biológica e social na periculosidade infantil.

Palavras-chave: infância; maternidade; delinquência; pobreza.

\footnotetext{
* Doctora en Ciencias Sociales Universidad Nacional de Buenos Aires. Investigadora asistente en el Instituto Patagónico de Estudios de Humanidades y Ciencias Sociales, Consejo Nacional de Investigaciones Científicas y Técnicas - Universidad Nacional del Comahue. E-mail: marianadovio@yahoo.com.ar.

(D) https://orcid.org/0000-0001-9209-1568
} 


\title{
Why do our children commit crimes? Childhood and criminological publications
} (Buenos Aires, 1930-1946)

\begin{abstract}
Around 1930 in Buenos Aires, physicians and lawyers began asking why children were committing crimes as part of a penal agenda revealed in publications such as the Revista de Psiquiatría, Criminología y Medicina Legal and the Anales de la Sociedad Argentina de Criminología from 1930 to 1946. Emerging from this discourse, conceived as a social practice, was an approach to the dangerous nature of childhood, linked to biological qualities, environmental factors and issues related to family and gender which were flagged as a precursor to crime. Advances in endocrinology, criminal biotypology, and psychiatry were resumed, with these subjects also addressed at the First International Congress on Criminology in Rome and the First Latin American Congress on Criminology in Buenos Aires, both held in 1938. A qualitative analysis of the discourse reveals a blend of the incidence of the biological and the social in the dangerous nature of childhood.
\end{abstract}

Keywords: childhood; maternity; crime; poverty.

\section{Pourquoi nos enfants deviennent-ils des délinquants? Enfance et publications criminologiques (Buenos Aires, 1930-1946)}

\section{Résumé}

Dans les années 1930 à Buenos Aires, des médecins et des avocats se sont demandé pourquoi les enfants pouvaient devenir des délinquants dans le cadre de recherches criminelles que l'on peut consulter dans des publications telles que Revista de Psiquiatría, Criminología y Medicina Legal et Anales de la Sociedad Argentina de Criminología entre 1930 et 1946. À partir de ces discours, conçus comme une pratique sociale, a été abordée la dangerosité de l'enfance en lien avec des spécificités biologiques et des aspects environnementaux, familiaux et de genre décrits comme des terrains propices au délit. On se basait alors sur les avancées de l'endocrinologie, de la biotypologie criminelle et de la psychiatrie. Ces thématiques ont également été abordées à l'occasion du Premier Congrès international de criminologie de Rome et du Premier Congrès latino-américain de criminologie de Buenos Aires, tous deux tenus en 1938. Sur la base d'une analyse qualitative du discours, nous avons identifié que l'on défendait à l'époque un mélange d'incidence biologique et sociale dans la dangerosité de l'enfance.

Mots-clés : enfance ; maternité ; délinquance ; pauvreté.

\section{为什么我们的孩子会犯罪？童年和犯罪学出版物 (布宜诺斯艾利斯，1930-1946)}

\section{摘要}

1930 年代前后，在布宜诺斯艾利斯，医生和律师都想知道为什么有儿童犯罪，这是犯罪学议题的一部分，这些议题在 1930 年至 1946 年间出现在《精神分析学、犯罪学、与法医学杂志》(Revista de Psiquiatría、Criminología y Medicina Legal) 和《阿根廷犯罪学会年刊》(Anales de la Sociedad Argentina de Criminología) 等出版物中。在这些期刊发表的 话语中，儿童犯罪学被视为一种社会实践，童年的危险性得到了探讨。当时的专家们普遍认为，儿童犯罪与生物特 质、环境、家庭和性别方面有关，这些方面被认为是犯罪前的场景。在内分泌学、犯罪 - 生物类型学和精神病学取得 进展的同时，儿童犯罪学也得到了发展。儿童犯罪学这些问题在 1938 年在罗马举行的第一届国际犯罪学大会和在布 宜诺斯艾利斯举行的第一届拉丁美洲犯罪学大会上得到了探讨。本文对那时期的一些话语进行了质性分析，发现它们 都把生物学的观点与社会学知识结合在一起，认为这两方面共同决定了儿童犯罪的发生，及其危险性。

关键词：童年；妇产科；违法行为；贫困。 


\section{Passagens. Revista Internacional de História Política e Cultura Jurídica \\ Rio de Janeiro: vol. 14, $\mathbf{n}^{0}$ 1, janeiro-abril, 2022, p. 89-110.}

\section{Introducción}

En 1935 el pedagogo Ernesto Nelson ${ }^{1}$ tituló un artículo de su autoría en la reconocida Revista de Criminología, Psiquiatría y Medicina Legal “¿Por qué delinquen nuestros niños?” Esta pregunta era parte de una agenda penal construida por médicos y abogados en Buenos Aires que incluyó clasificaciones criminológicas y psiquiátricas, instituciones de justicia para menores y prevención de la transgresión infantil. En esta oportunidad, nos detendremos en el abordaje, desde discursos expertos, de la peligrosidad en la niñez, categoría que incluyó cualidades, en algunos casos, consideradas inmanentes al cuerpo o psiquis que remitían a la potencialidad de comportamientos estimados riesgosos en términos morales $y$, no necesariamente, infracciones o delitos en términos jurídicos (DOVIO, 2019; FERLA, 2012). Su importancia radicó en que fue considerada un escenario previo al delito que debía ser identificada y generar mecanismos para prevenirlo.

Para este trabajo nos ubicamos dentro de las elaboraciones que estudian a la niñez como un colectivo específico que no consigna una fase evolutiva natural sino una construcción social caracterizada por su contingencia, historicidad y relación con la política y la cultura (BRIOLOTTI, 2021; ZAPIOLA, 2009). En las primeras décadas del siglo la representación dominante de la niñez en Buenos Aires fue vista como individuos dependientes, tanto física como emocionalmente, y necesitados de protección, vigilancia y educación. Argentina compartió con el contexto internacional, durante el siglo XX, la asunción de un modelo de justicia tutelar que supuso un proceso de criminalización de la pobreza y que llevó a separar el concepto de niños y el de menores. Éstos eran los que debían ser socializados desde dispositivos legales y tutelares y estaban fuera del circuito familiar y escolar, y, los niños, que sin requerir estas intervenciones estaban bajo la autoridad de los padres. Este paradigma reprodujo criterios criminológicos del positivismo al establecer una relación determinista entre delincuencia, marginalidad y pobreza, confundiéndose la materia asistencial con la penal (BERTOLÉ; TORROBA, 2020, p. 24). Se identificaron los niños que estaban en peligro porque

\footnotetext{
${ }^{1}$ Ernesto Nelson (1873-1959) fue inspector y director de Colegios secundarios. Fue un activo propagandista de la cultura y educación norteamericanas y especialmente de la obra de John Dewey. Integrante de la Liga Nacional de Educación y Asociación por los derechos del niño y dirigente de las tres asociaciones más vinculadas a la difusión de americanismo en Argentina como el Rotary Club, Asociación Cristiana de Jóvenes y el Instituto de Cultura Argentino - norteamericano.
} 
habían sufrido abandonos y no habían recibido cuidados e instrucción, y, niños peligrosos con posibilidades de entrar en el delito (RODRÍGUEZ LÓPEZ, 2012).

Desde la bibliografía especializada se ha establecido que en la década de 1930 en Buenos Aires hubo un proceso de estructuración ideológica e institucional en torno a la niñez. Esto fue corporizado en una creciente centralización de las acciones estatales de tutela y protección, así como de creación de Tribunales de Menores y profesionalización de la asistencia social (FARIAS-CARRACEDO; ROCÍO JUÁREZ, 2018). ${ }^{2}$ Desde prácticas judiciales se tendió a negar la responsabilidad penal por comportamientos delictivos, en tanto que eran entendidos en función de dinámicas familiares. Los principios del modelo de las cortes juveniles de Chicago, de principios del siglo XX, que habían pretendido avanzar sobre los niños considerados pre - delincuentes, se difundieron tempranamente en Argentina (STAGNO, 2020, p. 163). En este modelo de justicia tutelar fue central el papel de los informes de médicos y visitadores sociales para las decisiones judiciales respecto de los niños. Por entonces, las miradas médicas descubrieron en los niños, especialmente de sectores populares, padecimientos, anormalidades y deficiencias en su desarrollo mental, así como tendencias a cometer delitos. En este sentido, la delincuencia infantil se vinculó estrechamente a la enfermedad (SOSENSKY, 2008). La infancia tutelada resultó en algunos casos, a la vez, medicalizada (CESANO, 2015). Las propuestas en Argentina desde la corriente de la higiene mental de reconocidos psiquiatras como Telma Reca, Lanfranco Ciampi y Gregorio Bermann tuvieron en común la creencia en que la detección temprana de desórdenes psíquicos podía resolver problemas como la delincuencia. Estos autores, como señala Cesano, introdujeron el abordaje de la categoría de niñez anormal que implicó una ruptura epistemológica con el alienismo tradicional. Aunque se ha señalado que en el caso de Argentina esto fue gradual, ya que, aún muy adentrados los años 30, psiquiatras continuaron vinculando la delincuencia infantil a la degeneración (CESANO, 2015, p. 138). En su lugar, se propuso la noción de

\footnotetext{
${ }^{2}$ En Buenos Aires en 1933 se realizó la Primera Conferencia Nacional de la "Infancia abandonada y delincuente" convocada por el Patronato Nacional de la Infancia, cuyas conclusiones influenciaron a los especialistas y políticos en la creación de la Dirección de Maternidad e Infancia (1936) y en la configuración de los Tribunales de Menores de la provincia de Buenos Aires (creados en 1937 por ley 4664), Santa Fe, Mendoza, San Juan y Córdoba que se fundaron en 1939, 1940, 1947, 1957, respectivamente (STAGNO, 2020, p. 191). Este desarrollo institucional tuvo dificultades para su puesta en marcha por falta de presupuesto. A nivel académico la niñez también fue una preocupación específica y existieron publicaciones como Revista Patronato de la Infancia.
} 


\section{Passagens. Revista Internacional de História Política e Cultura Jurídica}

Rio de Janeiro: vol. 14, $\mathbf{n}^{0}$ 1, janeiro-abril, 2022, p. 89-110.

constitución, cuya configuración resultaba de exhaustivos análisis médicos y ambientales, como estudiamos a continuación.

A nivel latinoamericano, se pueden identificar tendencias que aportan a la comprensión de la niñez como un constructo histórico y sociocultural producto de distintos saberes, discursos y prácticas (HERRERA; CÁRDENAS PALERMO, 2013, p. 290). Desde Colombia, Pachón (2007) prestó atención a la transición de la categoría de niños delincuentes a menores infractores, como señal de tensión entre la delimitación moderna del niño como frágil, y los discursos jurídicos que penalizaban a los que no se insertaban en el ámbito social. En el caso del México posrevolucionario, Sosensky (2008) estableció que en la justicia para menores se volvió central el papel de la pediatría, psicología y asistencia social. Asimismo, se estudiaron las costumbres y formas de recreación de los niños que vivieron en la calle (LECHUGA HERRERO, 2021). Desde Brasil se ha señalado un profundo proceso de criminalización de la pobreza en un modelo tutelar de justicia infantil (AMICH ELÍAS, 2007).

Estos antecedentes abordaron la delincuencia en la niñez desde discursos institucionales, judiciales y educativos, pero existen escasas referencias a elaboraciones específicas del campo de discursos expertos criminológicos. Su importancia radica en que los mismos fueron parte importante del basamento teórico analítico en la construcción de mecanismos de exclusión institucionales y de encauzamiento social, de niños que delinquían y de los considerados peligrosos o potenciales delincuentes. Asimismo, se generaron intercambios intelectuales sobre estos temas y recepción de ideas foráneas.

El objetivo del trabajo es estudiar la peligrosidad en la niñez desde publicaciones académicas y su circulación internacional, que prestaron atención a las causas que llevaban a los niños al delito. Como fuentes de indagación retomamos a Revista de Criminología, Psiquiatría y Medicina Legal y Anales de la Sociedad Argentina de Criminología en Buenos Aires entre 1930 y 1946. Ambas publicaciones fueron dirigidas por el médico psiquiatra Osvaldo Loudet ${ }^{3}$ y editadas desde los Talleres Gráficos de la Penitenciaria Nacional en Buenos

\footnotetext{
${ }^{3}$ Desarrolló una amplia acción universitaria en el campo de la medicina, psiquiatría y criminología argentinas. Titular de la cátedra de Psiquiatría en la Universidad Nacional de la Plata y conocido por su tesis La pasión en el delito, adquirió experiencia clínica en el Hospicio de las Mercedes. Dentro de sus obras se encuentra "El Padre Castañeda", "A la luz de la psicología patológica", "Más allá de la clínica" y "Médicos argentinos", entre otras. Sustituyó desde 1927 a Helvio Fernández en la dirección del Instituto de Criminología que funcionó dentro de la Penitenciaría Nacional.
} 


\section{Passagens. Revista Internacional de História Política e Cultura Jurídica}

Rio de Janeiro: vol. 14, ${ }^{0}$ 1, janeiro-abril, 2022, p. 89-110.

Aires. Configuraron fuentes de referencia para profesionales y expertos del período ligados a la medicina y al derecho. Circularon en espacios académicos como la Facultad de Medicina y la Facultad de Derecho de la Universidad de Buenos Aires, así como, en el ámbito de la Penitenciaría Nacional. También tuvieron difusión en espacios académicos de Brasil, Chile, Bolivia, México, Italia, Bélgica y España, entre otros.

Para estudiar sus discursos utilizamos una metodología cualitativa, entendiendo al último no como una mera relación lingüística sino como un entramado de enunciados que circulan entre los cuerpos en prácticas que son siempre relaciones de poder que constituyen sentido. La constitución de sentido brota no solo de qué dice sino de quién, a quién, dónde, desde qué posición de poder y en qué entramado. Esto permite pensar en la materialidad del lenguaje, expresada en las prácticas (MURILLO, 2008, pág. 41).

Revista de Criminología, Psiquiatría y Medicina Legal fue parte de una larga tradición académica del positivismo criminológico ya que fue continuación ininterrumpida de Archivos de Psiquiatría, Criminología, Medicina Legal y Ciencias Afines fundada por José Ingenieros en 1902 a partir de la cual se difundió el modelo de psicopatología criminal. Anales de la Sociedad Argentina de Criminología fue fundada en 1935 para la divulgación de actividades y trabajos realizados por miembros de la Sociedad Argentina de Criminología que tuvo como principal objetivo el estudio del individuo en estado peligroso. En especial, se difundieron comunicaciones de congresos sobre criminología. El corte temporal en 1946 para este trabajo se debe a la llegada del peronismo y a diversas modificaciones a nivel criminológico.

Como principal hipótesis, desde estos discursos, la peligrosidad habría sido asociada a la necesidad de la prevención del delito y de políticas sociales desde los primeros momentos de vida del niño. Se habría mixturado la incidencia de lo biológico y de lo ambiental en la configuración de la peligrosidad en la niñez, a partir de experiencias extranjeras y construcciones locales. Además, su delimitación conceptual habría tenido implicancias de género ya que lo que fue considerado dentro de esta categoría, en algunos casos, quebraba representaciones sobre los roles asignados a las mujeres, ligados a las actividades domésticas y de crianza.

En lo que sigue, estudiamos la incidencia del factor biológico en la peligrosidad en la niñez en Revista de Criminología, Psiquiatría y Medicina Legal y Anales de la Sociedad Argentina de Criminología. En particular, en relación a la biotipología y endocrinología criminal desde los trabajos de articulistas de Brasil, Chile e Italia. Luego, analizamos el papel de los 
factores sociales en la etiología de la peligrosidad e implicancias de género. Por último, estudiamos la peligrosidad infantil en el Primer Congreso Latinoamericano y el Primer Congreso Internacional de Criminología, celebrados en 1938 en Buenos Aires y Roma, respectivamente, a través de sus relatorios.

\section{Discursos criminológicos en Argentina y experiencias extranjeras}

Revista de Criminología, Psiquiatría y Medicina Legal fue uno de los íconos de la producción criminológica argentina, de amplia difusión internacional que contribuyó a la creación de una red de saberes latinoamericanos. Tuvo una larga trayectoria que se inició con José Ingenieros en 1902 con Archivos de Psiquiatría, Criminología, Medicina Legal y Ciencias Afines. A partir de 1914 dirigieron la continuación de esta publicación, llamada Revista de Criminología, Psiquiatría y Medicina Legal, Helvio Fernández y desde 1927 hasta 1950 Osvaldo Loudet.

Estas revistas, junto con Anales de la Sociedad Argentina de Criminología, fueron representativas de la matriz positivista criminológica que asoció el comportamiento infantil al atavismo que conducía a la delincuencia como parte de la no superación patológica de algún momento anterior de la marcha evolutiva (FERLA, 2012). Los pedagogos Víctor Mercante y Rodolfo Senet representaron, a principios de siglo, una postura cercana a Lombroso con sus intentos por identificar rasgos atávicos o primitivos en los niños desde su trabajo en la sección de pedagogía en la Facultad de La Plata que fueron publicados en Archivos. En éstos también se publicaron trabajos que indagaron por costumbres y hábitos sociales de niños en condiciones de pobreza, desde un positivismo de corte sociológico. Este fue el caso del trabajo de José Ingenieros en 1904 sobre niños vendedores de diarios que habían sido detenidos en el Servicio de Observación de Alienados de la Policía de la ciudad de Buenos Aires. El interés por la delincuencia en relación a la niñez continuó en Revista de Criminología, Psiquiatría y Medicina Legal, a partir de trabajos como los de José María Paz Anchorena "Prevención de la delincuencia en menores" de 1918, el texto de Carlos de Arenaza4 "El asilo policial" en el que

\footnotetext{
${ }^{4}$ Fue médico de la Policía de la Capital entre 1901 y 1922 como también de la Oficina Médico Legal de a Prisión Nacional entre 1905 y 1917. Difundió en el ámbito local experiencias institucionales y los cuerpos legales sobre menores abandonados y delincuentes en Inglaterra. En 1929 publicó las crónicas de sus viajes a Europa para dar a conocer las experiencias extranjeras que, en su parecer, debían fundamentar las intervenciones estatales
} 


\section{Passagens. Revista Internacional de História Politica e Cultura Jurídica}

Rio de Janeiro: vol. 14, $\mathbf{n}^{0}$ 1, janeiro-abril, 2022, p. 89-110.

revisó 70 historias clínicas de niños alojados en el Depósito de Contraventores de la policía de Buenos Aires. De Arenaza, hacia 1925, a través de su cargo en la Oficina Médico Legal de la Penitenciaría Nacional y la Alcaldía de Menores, había intentado demostrar que la delincuencia y la reincidencia eran rasgos distintivos entre aquellos que vivían o desarrollaban oficios callejeros (RODRÍGUEZ LÓPEZ, 2012, p. 185).

Hacia los años 30 la impronta de Revista de Criminología, Psiquiatría y Medicina Legal fue médico psiquiátrica con un perfil academicista en la que se recibían trabajos extranjeros, se difundían conferencias y actividades de algunas dependencias del Estado, así como, partes de libros y fallos judiciales. Participaron articulistas que trabajaron en espacios ligados a la atención de la niñez como el médico Carlos De Arenaza, el pedagogo Ernesto Nelson y el médico Adolfo Luis Poncet (médico legista y de los Tribunales de Menores de la Provincia de Buenos), Telma Reca ${ }^{5}$ y Carolina García Tobar, entre otros. También desde el extranjero publicaron sobre estos temas Ricardo Luna Vega de la cátedra de Criminología a cargo del médico Carlos Bambarén de Lima, Perú, Enrique Roxo y Leonidio Ribeiro desde Brasil, Julio Altman Smythe de Chile y se publicó el Código del Niño de Venezuela.

En estos discursos especializados, en países de Latinoamérica, la versión proveniente de Lombroso que asoció el comportamiento infantil delictivo al atavismo, fue relativizada a partir de las disputas desde diferentes disciplinas (como la psicología, psiquiatría, biotipología, entre otras) por generar predominancias en clave científica social (FERLA, 2005). Una de las principales fue la psiquiatría, aunque en los estudios criminológicos no se descartó ninguna información médica del individuo estudiado por ser la época de la ciencia del constitucionalismo. Elaboración proveniente de Italia y definido por el médico Giacinto Viola, maestro de Nicola Pende, como un programa biológico-social de mejora de la estirpe, en base a un exhaustivo análisis psico-somático de las personas para ubicar a cada individuo adecuadamente dentro de la división de roles de la sociedad (VALLEJO; MIRANDA, 2004, p.

en materia de la protección y reeducación de menores en Argentina. Inglaterra aparecía como un ejemplo por la paulatina moderación en las penas (STAGNO, 2020, p. 348).

${ }^{5}$ Telma Reca nació en 1903 y se graduó de médica en la Universidad de Buenos Aires. En 1930 obtuvo la beca otorgada por el Vasar Collegue de Nueva York a través del Instituto Cultural Argentino para estudiar cuestiones relativas al bienestar e higiene infantil. Volvió al país con la intención de crear un ámbito de trabajo similar al observado en las Child Guidance Clinics. En 1934 comenzó a trabajar en un consultorio que se llamó de higiene mental en el Hospital de Clínicas, dependiente de la cátedra de Pediatría del profesor Mamerto Acuña (TALAK, 2005, p. 591). 
432). La biotipología se situó dentro de esta corriente y su recepción en Argentina hacia 1930, mayormente a partir de Nicola Pende ${ }^{6}$ fue resultado de una larga tradición del pensamiento científico en América Latina que estuvo marcada por la temprana adhesión a los principios lamarckianos, basados en la herencia de características adquiridas y patologías obtenidas en el medio social como el alcoholismo, tuberculosis o sífilis que se podían transmitir a la descendencia provocando degeneración social (ERASO, 2007). La biotipología apareció como una apuesta para superar y complejizar la mirada antropológica prestando atención a múltiples facetas que incluyeron las características físicas, modalidades psíquicas y modulaciones morales de los cultores del delito (ROSSI, 1943).

La infancia, desde las teorías constitucionalistas, era un estadio especialmente interesante para realizar pruebas en los términos de un laboratorio y como paradigma para toda la cuestión de la criminalidad y la defensa social (FERLA, 2012, p. 110). A ello respondió, por ejemplo, la existencia de laboratorios de biología infantil para estudiar causales de delitos o formas anticipatorias de los mismos desde el estudio del cuerpo de los niños. Hubo diferentes modelos como el Laboratorio de Criminología de Madrid en los que participaron reconocidos profesionales como Bernaldo de Quirós, Salillas y Simarro a principios del siglo XX. En América Latina una experiencia fue el laboratorio de biología infantil fundado por Leonidio Ribeiro en 1936 en Rio de Janeiro e inspirado por el Centro Médico Pedagógico de Observación de Roma, que había surgido dos años antes. También existió una Sociedad Internacional de Biología Criminal que hacia 1933 recomendó la creación de institutos de biotipología criminal en América Latina (ROSSI, 1943, p. 178). Según Ribeiro (1936, p. 445):

\footnotetext{
En todos los países hay un movimiento de opinión, y en particular, en los médicos, con el fin de interesar a los poderes públicos en favor de la creación de centros de estudios biológicos de la niñez y la juventud, para descubrir precozmente sus anormalidades físicas y mentales, Rougier, Heuyer, Gilbert - Robin, Toulouse y Roubinovitch, en Francia, Vervaeck y Vermeylen en Bélgica, Di Tullio en Italia, Ruiz Funes, Jiménez de Asúa y Saldaña en España han insistido en que es la única forma de la verdadera prevención criminal.
}

En el marco de una sesión de la Sociedad Argentina de Criminología del 31 de octubre de 1936, Leonidio Ribeiro, difusor de la biotipología en Brasil, se refirió a la revelación de

\footnotetext{
${ }^{6}$ Fue un médico impulsor de la medicina constitucional y la biotipología en vinculación con el poder político de Mussolini.
} 


\section{Passagens. Revista Internacional de História Política e Cultura Jurídica \\ Rio de Janeiro: vol. 14, $\mathbf{n}^{0}$ 1, janeiro-abril, 2022, p. 89-110.}

anomalías en los niños desde el punto de vista de la prevención criminal. Para ello dio cuenta de la experiencia que estaba llevando a cabo en su laboratorio de biología infantil y que, en base a un informe por trabajos realizados en el mismo, había recibido en Turín el "Premio Lombroso". Ribeiro lo describió como un verdadero centro de observación destinado a demostrar que entre los niños abandonados y delincuentes existía un gran número de enfermedades generales y de anomalías. Esta iniciativa solo fue una institución modelo, cuyos efectos radicaron mayormente en la creación de imaginarios que estigmatizaron la delincuencia en potencia (FERLA, 2005).

Ribeiro (1936, p. 447) se refirió al difuso dominio de la peligrosidad al establecer: "creo, por lo tanto, que el laboratorio de biología infantil, podría ante todo revelar precozmente las anomalías de los menores delincuentes y, lo que es más sutil, lo que pueden llegar a ser". A partir de aquí se podía ahondar en el estudio del sustrato biológico, inclusive el rol de los desequilibrios glandulares. El metabolismo basal y la interferometría eran objeto de investigaciones y el laboratorio contaba con la aparatología para ello. Según Ribeiro (1936, p. 447 ), las primeras conclusiones de observaciones resultaban sorprendentes y dolorosas ya que "el cien por ciento estaban enfermos y no había un solo menor en perfecto estado de salud ya fuera por sífilis, epilepsia, parálisis general juvenil, tuberculosis y afecciones de la garganta y de la boca". Por medio del examen médico sistemático de los niños consideró que era posible identificar la constitucional delincuencial, especie de actualización del criminal en sintonía con lo que propuso el médico italiano Benigno Di Tullio, presidente por entonces de la Sociedad Internacional de Criminología, quien revalorizó, aún hasta la década del 30 , los avances de Lombroso. Di Tullio había aplicado la biotipología a la criminología diferenciando los factores del delito en tres causas; predisponentes, preparatorias y desencadenantes. Su mérito, según Arturo Rossi, había sido propiciar las relaciones de la criminología con la antropología, la bio fisio - psicología, eugenesia y medicina constitucional. En su instituto en Roma Di Tullio estudió la etiología del delito y desenvolvimiento de varias tendencias y aptitudes criminosas a partir de la personalidad, antecedentes hereditarios, fórmula glandular endócrina y características antropológicas y psiquiátricas. La constitución delincuencial representó la condición potencial favorable al surgir del hecho delictuoso, el que podría manifestarse o no, según circunstancias accidentales capaces de provocarlo (ROSSI, 1943, p. 179). En el Primer Congreso Internacional de Criminología Di Tullio había propuesto distinguir entre: menores delincuentes 


\section{Passagens. Revista Internacional de História Política e Cultura Jurídica}

Rio de Janeiro: vol. 14, ${ }^{0} 1$, janeiro-abril, 2022, p. 89-110.

comunes (moralmente abandonados o pseudo - anormales) y menores delincuentes ocasionales. Dentro de éstos distinguió predispuestos constitucionalmente al delito y anormales psíquicos con reacciones criminosas episódicas (LOUDET, 1939, p. 21).

En el centro de Ribeiro hubo asistentes sociales para determinar cuál era la modalidad de la vida familiar de los niños. Cada asistente hacía un resumen del resultado de su investigación que era remitida a un tribunal, indicándose el método de tratamiento y formulando consejos, desde el punto de vista de la educación, de tal manera que la colaboración del juez con el médico y pedagogo fuera estrecha. En 1938 Ribeiro participó en Buenos Aires en las Segundas Jornadas Biotipológicas estableciendo que era posible clasificar biotipológicamente la primera infancia y desde los primeros balbuceos a todos los que por su constitución pudieran ser considerados pre - delincuentes, pasibles de tratamientos para atenuar o corregir sus anomalías y consecuentes reacciones antisociales. Fue invitado y atentamente escuchado por Arturo Rossi, representante de la biotipología en Argentina, quien coincidía con los beneficios de esta corriente, aunque las fichas orto-genéticas escolares por él creadas solo fueron parte de una experiencia piloto en la provincia de Buenos Aires. Estas no tuvieron fines específicamente criminológicos, sino escolares y de "cuidar el desarrollo óseo y muscular, la agilidad y destreza de los movimientos, mejorar el terreno de los escolares y hacer que todos crezcan sanos, fuertes y productivos" (ROSSI; GRAFIGNA, 1938, p. 11).

Una parte importante dentro de la biotipología fue la endocrinología ya que las glándulas de secreción interna actuaban en la constitución del individuo, su fisiologismo, vida humoral, temperamento y vida. Altman Smythe (1934, p. 420) desde Chile estableció "Pende ha dicho que de la fórmula endócrina individual depende la personalidad psíquica de todo sujeto". El anormal funcionamiento del aparato endócrino del niño podía hacerle adquirir peligrosidad. El niño podía ser peligroso antes de nacer porque:

La peligrosidad del menor puede ser adquirida en el vientre materno, también en el momento de la concepción, cualquier intoxicación, cualquier trastorno de las glándulas endócrinas, en fin, cualquier desequilibrio orgánico que se produzca en la madre, puede repercutir en el menor, dando lugar a una transformación física y psíquica tal, que lo convierta en un ser orgánicamente peligroso (SMYTHE, 1934, p. 416).

En esta misma línea, el profesor Enrique Roxo, (titular de clínica psiquiátrica de la Universidad de Rio de Janeiro y director del Instituto de Psicopatología) estableció que el estudio de la endocrinología ayudaba a distinguir a los niños anormales peligrosos: 
Es la forma endócrina anormal la que influye en su personalidad. El mal funcionamiento de la glándula pineal, perturba en los niños el desarrollo normal de los caracteres sexuales secundarios y da la tendencia a practicar actos contra el pudor $u$ otros delitos de esta naturaleza. Dícese que el hiper- funcionamiento de las glándulas de secreción interna en la primavera explica la razón de ser de muchas actividades criminosas en esta estación. Pende y Marañón señalan que el sol, la humedad, la presión atmosférica, el mar, etc. influyen en la tendencia al crimen, actuando sobre las glándulas de secreción interna, principalmente tiroidea y supra - renales (ROXO, 1936, p. 499).

\section{Etiología social de la peligrosidad en discursos criminológicos. Implicancias de género y prevención del delito}

En Revista de Criminología, Psiquiatría y Medicina Legal, el médico Ricardo Luna Vega (1941, p. 17), desde Perú, estableció que "para resolver el problema de la peligrosidad en los menores debe empezarse por ampliar las bases de la economía nacional para elevar el nivel de vida de las clases populares". Según Luna Vega (1941, p. 14), que trabajó junto a Carlos Bambarén, en Perú no había prácticas ni estadísticas sobre las causas de la peligrosidad, sin embargo, ese estudio debía hacerse, "ya que el conocimiento de las condiciones biológicas y mesológicas es la etapa orientadora para un verdadero régimen tutelar".

La peligrosidad en la niñez tuvo como uno de sus principales carriles de expresión el estudio de las causales sociales del delito, que se complementaban con los datos sobre aspectos biológicos, $y$, en algunos casos, lo reforzaban. Muchas veces era un ambiente social hostil el indicado como favorable de comportamientos peligrosos. La dimensión biológica, que facilitaba la peligrosidad, coexistió con tensiones y relaciones de colaboración, con lo ambiental y lo psíquico. En términos jurídico- institucionales, las competencias que fueron establecidas para los jueces de menores fueron amplias y contemplaron la situación social y familiar de los niños. A través de la ley 4.664 , de creación de Tribunales de Menores en la provincia de Buenos Aires, se estableció su competencia respecto de menores de dieciocho años en los casos que fueran autores o cómplices de un delito, por faltas o contravenciones, abandonados o en peligro moral, si infringían disposiciones legales sobre instrucción o trabajo y en cualquier otro caso en que afectaran sus personas por orfandad o abandono. Además, cuando por actos reiterados de inconducta obligaban a los padres o tutores a recurrir a la autoridad judicial y por faltas o contravenciones cometidas por mayores que afectaran a menores. Desde esta ley, se incorporó una ideología específica sobre el patronato por la cual se igualó al menor abandonado y al delincuente y se incorporaron los conceptos de peligro moral y material. La 


\section{Passagens. Revista Internacional de História Politica e Cultura Jurídica}

Rio de Janeiro: vol. 14, $\mathbf{n}^{0}$ 1, janeiro-abril, 2022, p. 89-110.

finalidad primordial del tribunal de menores en Buenos Aires fue comprender el origen del delito y fue común la judicialización de situaciones como borracheras, peleas y disturbios en la calle (STAGNO, 2020, p. 192). También se definieron los roles del médico y visitadores sociales que podían justificar, en algunos casos, a los niños con debilidades como potenciales delincuentes. Adolfo Luis Ponce (1950, p. 306), desde su labor como médico de tribunal de menores, estableció respecto de la obra de los mismos en la década de 1930:

La obra de visitadores es de innegable importancia. Son ellos los ojos inteligentes del tribunal que se filtran en la vida familiar y recogen la impresión acabada del ambiente en que actúa el menor cuya conducta ha comenzado a preocupar a propios y extraños. Sus observaciones son de valor en el estudio de la etiología de la delincuencia infantil, indicando que los casos de abandono son generalmente explicados por la desintegración de los hogares en principio mal constituidos

Otra de las articulistas que prestó atención a la peligrosidad partiendo de la relevancia del ambiente y presupuestos de la medicina constitucional fue Telma Reca, quien publicó en 1932 "El estudio social del niño delincuente" y "Concepto de la delincuencia infantil" en Revista de Criminología, Psiquiatría y Medicina Legal. Este trabajo fue parte de su tesis doctoral Delincuencia infantil en los Estados Unidos y en la Argentina en la que estableció que existían síntomas que podían anticipar delitos y debían ser estudiados:

Es fácil el estudio de la situación social del niño y el conocimiento de los casos en que debe ejercerse el patronato. Es, en cambio, difícil reconocer los síntomas pre delictuales, o precisar qué debe entenderse por ellos. Sin embargo, la extensión del concepto de delito infantil a los síntomas que pueden preludiarlo exige este estudio y determinación (RECA, 1932).

La elaboración de Reca (1932) dio cuenta de los esfuerzos por delimitar conceptualmente la noción de la peligrosidad a partir de síntomas psíquicos que pudieran ser detectados de forma precoz. Dentro de las influencias sociales Reca destacó, por ejemplo, la constitución de la familia ya que si ella se desintegraba no era posible que tuviera el papel esperado para la formación del niño. También mencionó las dificultades de padres y, sobre todo, de madres para asistir y educar a sus hijos cuando volvían con cansancio de sus trabajos. Como elementos de incidencia en la conducta del niño señaló la pobreza y diversas expresiones de la misma, la vivienda insalubre, hacinamiento y trabajo infantil. Reca asoció estas conductas a los sectores sociales pobres a tono con el ideario higienista decimonónico y planteó que era necesario brindar protección prenatal a la madre (RAMACCIOTTI, 2018, p. 182). Su manera de estudiar la peligrosidad como 


\section{Passagens. Revista Internacional de História Política e Cultura Jurídica \\ Rio de Janeiro: vol. 14, $n^{0}$ 1, janeiro-abril, 2022, p. 89-110.}

un objeto de estudio específico fue, en parte, a partir de extender el concepto de delincuencia infantil a la desadaptación social y a fallas en la educación familiar. Para ello partió de que existía un sustrato biológico permanente en el individuo y otro social en continua transformación que era tan variable como la vida misma (RECA, 1932, 157). De esta forma en su concepción psicopatológica anudó lo biológico y lo social.

Esta perspectiva que puso acento en el papel ambiental y familiar también estuvo representada por trabajos del educador Ernesto Nelson que presentó en las sesiones de la Sociedad Argentina de Criminología y luego publicó en Revista de Criminología, Psiquiatría y Medicina Legal el trabajo que tituló con la pregunta: “¿Por qué delinquen nuestros niños?”, mencionado al principio de este trabajo. A esto quería responder a partir de una lectura que realizó de 850 prontuarios penales y tomó en cuenta tres factores criminógenos que consideró decisivos: padres extranjeros con modalidades autoritarias de crianza (italianos, rusos, etc.), madre que trabaja y hogar degradado. En el caso del ítem madre que trabaja hizo alusión a efectos imaginados perniciosos en los niños a raíz de las que trabajaban en fábricas o fuera del hogar "La falta de la madre hemos dicho que es un factor que aparece en la familia del menor delincuente con mucha más frecuencia que en la familia normal" (NELSON, 1935, p. 854).

El ítem construido por Nelson también implicó una postura antifeminista en relación al modelo de familia tradicional y al rol del padre como proveedor económico. La vinculación de la ausencia de la madre a una posible futura delincuencia o peligrosidad de los hijos, formó parte de concepciones de género que se plasmaron en las publicaciones analizadas. Desde esa postura, la madre era la principal responsable de la crianza y el sostén emocional del hogar. Además, fue parte del proceso de inserción laboral de las mujeres durante el período de entreguerras y los debates que se generaron en torno a cuál era su rol en la sociedad, en el que preponderó su función maternal. Desde esta perspectiva, el mejor destino del niño en su primera y segunda infancia era estar con su madre presente durante todo el día. Esto también fue parte de la defensa del binomio madre e hijo en elaboraciones sobre la peligrosidad en la infancia. Este binomio refirió a un vínculo indisoluble que había que proteger desde múltiples mecanismos que fueron desde la lactancia, presencia de la madre en la crianza y dispensarios de lactantes, entre otros. Configuró, en Argentina, uno de los lineamientos de la protección materna infantil expresado en la ley 12.341 de creación de la Dirección de Maternidad e Infancia en 1936. 


\section{Passagens. Revista Internacional de História Politica e Cultura Jurídica}

Rio de Janeiro: vol. 14, $\mathbf{n}^{0}$ 1, janeiro-abril, 2022, p. 89-110.

Según Nelson, era más probable la entrada de los niños a la delincuencia si su madre los abandonaba que si lo hacía su padre. Sin embargo, resaltó que, ante el abandono del padre, la mujer por necesidad económica recurría a salidas llamadas más fáciles e inmorales como la prostitución. Esto fue abordado desde la producción científica pero también de la cultura de masas ya que el discurso literario reprodujo esta ideología en las representaciones de "el mal paso", la "caída", asociando el trabajo femenino con conductas sexuales incorrectas por momentos rayanas a la prostitución (QUEIROLO, 2011). El autor se refirió a los efectos de la orfandad parcial estableciendo que la falta de padre era un factor perturbador máximo en la vida del niño por verse la familia privada, en tal caso, del elemento que en ella concurre al sostén económico:

Sin embargo, de acuerdo a nuestras estadísticas, la falta de madre entre los abandonados y delincuentes ocurre 6,7 veces con más frecuencia que entre los niños de la población general, mientras la falta de padre aparece sólo 3.3 veces más. Querría decir esto que la falta de madre concurre a la delincuencia y abandono de los menores con una intensidad dos veces mayor que la falta de padre (NELSON, 1935, p. 852).

Ernesto Nelson en 1945 volvió a publicar sobre el tema refiriéndose "Hacia un programa coordinado de prevención de la delincuencia infantil" en Revista de Criminología, Psiquiatría y Medicina Legal. En éste realizó propuestas sobre modificaciones en el ambiente social y familiar. En especial, debe destacarse que se posicionó a favor de una protección integral de la infancia, entendiendo por ello que se debían iniciar acciones desde los primeros momentos de vida de los niños. Nelson (1945, p. 231) abogó por implementar políticas preventivas del delito que abarcaban no sólo la escuela, sino el hogar, el uso del tiempo de recreación, la asistencia psiquiátrica, mejoras económicas y sociales:

Toca al Estado, decíamos, complementar los reformatorios y asilos por instituciones genuinamente profilácticas y protectoras de todo niño expuesto a caer, más tarde o más temprano, en la desorganización social. Tales son, para la primera y la segunda infancia, esos democráticos centros de higiene maternal e infantil, los dispensarios de lactantes, los nidos, las casas cunas, las formas modernizadoras del viejo asilo maternal, los jardines de infantes del tipo de las nursery schools inglesas y de la moderna escuela maternal francesa.

Además, mencionó el caso de las dependencias llamadas "casas infantiles" surgidas por iniciativa del doctor Juan Carlos Lando, para la protección de la infancia de la provincia de Buenos Aires donde no llegaba la acción del juez, a la que acudían libremente las madres necesitadas del auxilio social que esas casas prestaban: la asistencia diurna al niño mientras 
ellas trabajaban. "En esas casas el niño se alimenta, juega y prepara sus trabajos para la escuela, a la que se encamina en grupo alegre" (NELSON, 1945, p. 232).

\section{EI Primer Congreso Internacional de Criminología y el Primer Congreso Latinoamericano}

El Primer Congreso Latinoamericano de Criminología fue organizado por la Sociedad Argentina de Criminología en Buenos Aires en julio de 1938, asistieron más de 600 invitados de la región y estuvo patrocinado por el Ministerio de Instrucción Pública a cargo del jurista Jorge Eduardo Coll. En los Anales de la Sociedad Argentina de Criminología se dedicó una sección a los trabajos de la delegación argentina que participó, y el médico Carlos de Arenaza en su intervención dio cuenta de que su preocupación no era sólo en torno a los delitos, sino a las reacciones antisociales en los niños, como paso previo a la delincuencia. En su comunicación aportó resultados estadísticos obtenidos en más 3.000 observaciones personales que había realizado en la Penitenciaría Nacional y la Alcaidía de Menores, entre 1904 y 1907, aunque se centró en 625 casos de niños que no estaban procesados por delitos. Identificó causas biológicas y sociales en las reacciones antisociales y manifestó dificultades que había tenido como médico para hallarlas, aún con el auxilio de psiquiatras, juristas y diversos profesionales que a lo largo de su extensa carrera había convocado en sus estudios. Entre los factores biológicos individuales, la deficiencia mental y el heredo - alcoholismo era lo que había encontrado con mayor frecuencia, y, en menor medida, algunas psicosis, neurosis y trastornos de importancia variable en la esfera mental y psíquica (DE ARENAZA, 1938).

El factor biológico era única causal de la delincuencia en un limitado número de casos ya que en la mayoría tenía peso el factor sociológico y el rol de la familia: "luego la delincuencia del menor es la consecuencia no ya de una sola causa sino de la concurrencia de varias" (DE ARENAZA, 1938, p.25). Estableció que existían dificultades respecto de la medición de los factores sociales en las causas de la delincuencia infantil, porque las estadísticas dentro de la Penitenciaría Nacional habían sido realizadas por médicos que sólo habían observado aspectos personales. Sin embargo, reconocía que las más sutiles influencias del hogar familiar como la capacidad o inteligencia de los padres, su compasión y trato mutuo, actitudes con los 


\section{Passagens. Revista Internacional de História Política e Cultura Jurídica \\ Rio de Janeiro: vol. 14, $n^{0}$ 1, janeiro-abril, 2022, p. 89-110.}

hijos y entre sí, eran factores decisivos y fundamentales respecto de los problemas de conducta del niño.

Según De Arenaza, las reacciones antisociales eran síntomas de dificultades en la familia, el hogar y ambiente que perturbaban el desarrollo del niño y tenían mayor eficacia si obraban sobre organismos preparados o debilitados por afecciones patológicas, adquiridas o transmitidas por sus progenitores. De ordinario no era posible distinguir entre factores sociales y biológicos y para encarar la lucha contra el abandono y la delincuencia, se debía partir de que las reacciones antisociales de los menores eran consecuencia de la desorganización de la familia.

Desde la delegación de Brasil, Pacheco y Silva (director del Manicomio Judicial de San Pablo) testimonió la misma indefinición respecto del énfasis en la tendencia ambiental y la orgánica en la etiología de la criminalidad en la niñez, aunque coincidieron en que era necesario internar a niños problemáticos (FERLA, 2005). En el cierre de este Congreso Jorge Eduardo Coll concluyó en que debían observarse tanto los factores biológicos como los sociales para combatir el delito infantil.

Tres meses después del Primer Congreso Latinoamericano de Criminología, en octubre de 1938, se llevó a cabo en Roma el Primer Congreso Internacional de Criminología y asistió una delegación argentina encabezada por Osvaldo Loudet como representante de la Sociedad Argentina de Criminología. Dentro de los temas del congreso se incluyó: "Etiología y diagnóstico de la criminalidad infantil e influencia de tales búsquedas sobre las providencias judiciales". Los relatores sobre este tema fueron P. De Casabianca, consejero honorario de la Corte de Casación de París, A. De Marsico, profesor de la Universidad de Nápoles y P. Pisani, profesor de la Universidad de Messina.

Uno de los principales debates fue en torno al peso de los factores endógenos y exógenos en las causas de la criminalidad infantil (LOUDET, 1939, p. 187). Se estableció que la distinción entre ambos era analítica ya que era ardua en muchos casos en el estudio clínico. Entre los autores que documentaron la preponderancia de los factores biológicos estuvo el Dr. Mennato que sobre doscientos menores del reformatorio judicial de Nisida encontró a $78 \%$ con herencia patológica (alcoholismo $54 \%$, criminalidad $43 \%$, sífilis, $12 \%$, tuberculosis $10 \%$ y psicopatías, $8,5 \%)$. En cambio, otros relatores dieron preeminencia al factor social como D. Pisani, de Italia, que, sobre 1.464 menores sometidos a estudio procedentes del centro de observación para menores en Roma, encontró $66,87 \%$ provenientes de familias desorganizadas, condiciones 


\section{Passagens. Revista Internacional de História Politica e Cultura Jurídica}

Rio de Janeiro: vol. 14, $\mathbf{n}^{0}$ 1, janeiro-abril, 2022, p. 89-110.

económicas malas, $76 \%$ analfabetos y $39,8 \%$ de herencia patológica. En este estudio la delincuencia morbosa quedo reducida al 20\% (LOUDET, 1939, p. 186).

En el Congreso se concluyó sobre la necesidad de futuras investigaciones sobre la etiología de la criminalidad infantil y de llegar a la unificación internacional de los métodos de investigación. Asimismo, de proceder según un criterio de clasificación común, tanto en las investigaciones como en el tratamiento a seguir en los menores antisociales. Se distinguió entre los niños que tuvieran patologías mentales o anomalías consideradas de base, desde el punto de vista biológico psíquico, que sería los considerados anormales. En otro grupo estuvieron los niños ligados a la peligrosidad que en general eran quienes, sin ninguna anormalidad detectada, cometían infracciones menores. En ambos congresos estudiados hubo esfuerzos por compatibilizar lo bio - social.

\section{Reflexión final}

Para finalizar, retomamos la importancia del estudio de los discursos expertos en criminología que prestaron atención a las causas que llevaban a los niños al delito. Algunas de estas elaboraciones reforzaron fundamentaciones de mecanismos de exclusión y también de encauzamiento social, tanto de niños que delinquían o peligrosos o potenciales delincuentes.

Como principal hallazgo del trabajo realizado establecemos que la peligrosidad en la niñez fue abordada de forma específica desde Revista de Criminología, Psiquiatría y Medicina Legal y Anales de la Sociedad Argentina de Criminología en Buenos Aires, entre 1930 y 1946 , y fue vinculada a comportamientos desadaptados, considerados el escenario previo al delito. Esto se expresó, mayormente, a través de la búsqueda de su etiología, distinguiendo entre factores biológicos y ambientales. Los primeros retomaron el cuerpo del niño como basamento para medirlo a través de la biotipología, en tanto construcción sofisticada de las elaboraciones de Lombroso. Esto fue a partir de experiencias y estudios extranjeros, como las de Leonido Ribeiro, Julio Altman Smythe y Enrique Roxo. La noción de un delincuente constitucional retomado por Ribeiro tomó como base las elaboraciones de Benigno Di Tullio que estableció, en el Primer Congreso Internacional de Criminología en Roma, que existían niños predispuestos constitucionalmente al delito. Se tomaron en cuenta los efectos comportamentales en los niños de los desórdenes endocrinológicos, la herencia neuropática, 


\section{Passagens. Revista Internacional de História Política e Cultura Jurídica \\ Rio de Janeiro: vol. 14, $\mathbf{n}^{0}$ 1, janeiro-abril, 2022, p. 89-110.}

alcoholismo, sífilis y tuberculosis. En algunos casos, la peligrosidad fue un término utilizado por psiquiatras para identificar patologías de base en niños institucionalizados o detenidos por infracciones menores.

También se tuvo en cuenta el papel de los factores sociales, incluso en las iniciativas de laboratorios de biología infantil, como la experiencia de Ribeiro, se contrató a visitadoras sociales para que indagaran en la dinámica familiar de los niños. En general, fue muy dificultosa la distinción de cuál era la incidencia de los factores biológicos y sociales en la delincuencia infantil. Por entonces, la etiología social tuvo predominancia en ámbitos judiciales y educativos en Buenos Aires y se judicializaron situaciones consideradas pre - delictuales como borracheras o disturbios. Igualmente, la relevancia de lo ambiental tuvo diversos desafíos porque eran difícilmente mensurables e identificables al remitir a múltiples y heterogéneas condiciones de vida y familiares (hacinamiento, pobreza, alcoholismo, trabajo infantil, entre otros). Esta postura estuvo representada por Telma Reca que estableció la necesidad de la detección precoz de la peligrosidad y la importancia del estudio de la familia. También adquirió relevancia el papel de las visitadoras sociales para la detección de conductas consideradas preocupantes en los niños.

En discursos criminológicos, el niño estimado peligroso fue asimilado, en parte, al desprotegido, en primera instancia por su familia o entorno más cercano, y, en segundo lugar, por el Estado. Ernesto Nelson indagó sobre las causas de la delincuencia infantil y las implicancias de género en ellas a través de su elaboración del factor criminógeno la madre que trabaja, señalando los posibles efectos adversos de su ausencia en el comportamiento del menor. Esta situación atentaba contra la preservación del binomio madre e hijo que había sido retomada como un lineamiento dentro de las políticas de protección a la infancia en Argentina por entonces. Desde estos discursos, la prevención de la peligrosidad debía ser desde la primera infancia a través de la tutela y la protección, siguiendo el ideario predominante y la institucionalización que hubo en este período a través de tribunales para menores, dispositivos de asistencia materno - infantil y profesionalización de la asistencia social. Las propuestas e iniciativas de protección coexistieron con posturas que buscaron en el niño, como potencial delincuente, un sustrato biológico que iba a determinar su vida futura. 


\section{Referencias}

AMICH ELIAS, Cristina. La evolución de la legislación sobre menores de edad delincuentes en la dictadura militar brasileña. Revista Latinoamericana de Ciencias Sociales, Niñez y Juventud, Manizales, v. 5, n. 2, p. 639-690, 2007. Disponible en: http://www.scielo.org.co/scielo.php?script=sci_arttext\&pid=S1692-715X2007000200006.

Acceso en: 22 oct. 2021.

BERTOLÉ, Cecilia; TORROBA, Esteban. Los "niños de la calle" en la observación general 21 del comité de los derechos del niño: transfiguración de una categoría de comprensión social. Revista Perspectivas de las Ciencias Económicas y Jurídicas, Santa Rosa, v. 10, n. 1, p. 1844, 2020. http://dx.doi.org/10.19137/perspectivas-2020-v10n1a02

BRIOLOTTI, Ana. La educación desde la cuna: psicología y orden social en la puericultura argentina (1934-1955). Espacio, Tiempo y Educación, Salamanca, v. 8. n. 1, p. 31-52, 2021. https://doi.org/10.14516/ete.350

CESANO, Daniel. Medicalizando la niñez delincuente, intervenciones psiquiátricas en la criminalidad infantil (Buenos Aires, Rosario, Córdoba, 1920-1940). Córdoba: Brujas, 2015.

DE ARENAZA, Carlos. Valoración de los factores biológicos y sociológicos en las reacciones antisociales de los menores. Anales de la Sociedad Argentina de Criminología, Buenos Aires, v. I, p. 23-42, 1938.

DOVIO, Mariana. Discursos desde la Sociedad Argentina de Criminología, Buenos Aires, 19331945. Revista Historia y Justicia, Santiago de Chile, v. 13, p. 1-26, 2019. https://doi.org/10.4000/rhj.3023

ERASO, Yolanda. Género y eugenesia. Hacia una taxonomía médico-social de las mujeresmadres en la década del treinta". En: BRAVO, María; GIL LOZANO, Fernanda; PITA, Valeria (Comp.). Historias de luchas, resistencias y representaciones: mujeres en la Argentina, siglos XIX y XX. Tucumán: Universidad Nacional de Tucumán, 2007. p. 361-390.

FERLA, Luis. El niño, el médico, el policía y el patrón. Infancia y determinismo biológico en el Brasil de entreguerras. En: MIRANDA, Marisa; VALLEJO, Gustavo (Comp.). Darwinismo social y eugenesia en el mundo latino. Buenos Aires: Siglo XXI, 2005. p. 401- 440.

FERLA, Luis. El determinismo biotipológico y su red de sustentación a través de eugenistas españoles, brasileños y argentinos. En: MIRANDA, Marisa; GIRÓN SIERRA, Álvaro (Coord.). Una Historia de la Eugenesia: Argentina y las redes biopolíticas internacionales 1912-1945. Buenos Aires: Biblios, 2012. p. 97-120.

FARIAS-CARRACEDO, Carolina; ROCÍO JUÁREZ, Ana. ¿Qué les Pasa a Estos Niños? Un estudio acerca de la incidencia de los saberes psi en la solución de las problemáticas de la infancia en Buenos Aires desde comienzos del siglo XX hasta 1940. Revista Psicologia e Saúde, Campo Grande, v. 10, n. 3, p. 57-70, 2018. https://doi.org/10.20435/pssa.v10i3.615 
HERRERA, Martha Cecilia; CÁRDENAS PALERMO, Yeimy. Tendencias analíticas en la historiografía de la infancia en américa latina. Anuario Colombiano de Historia Social y de la Cultura, Bogotá, v. 40, n. 2, p. 279-311, 2013. Disponible en: http://www.scielo.org.co/pdf/achsc/v40n2/v40n2a10.pdf. Acceso en: 22 agosto 2021.

LOUDET, Osvaldo. Primer Congreso Internacional de Criminología, Roma. Anales de la Sociedad Argentina de Criminología, Buenos Aires, v. 5, p.180-212, 1939.

LECHUGA HERRERO, Daniela. Entre el ocio y el trabajo: la infancia popular en las calles de Ciudad de México en la década de 1930. Trashumante: Revista Americana De Historia Social, Antioquía, v. 17, p. 198-218, 2021. https://doi.org/10.17533/udea.trahs.n17a09

LUNA VEGA, Ricardo. Factores etiológicos de la peligrosidad en los menores. Revista de Criminología, Psiquiatría y Medicina Legal, Buenos Aires, v. 8, p. 9-17,1941.

MURILLO, Susana. Producción de pobreza y construcción de subjetividad. En: CIMADAMORE, Alberto; CATTANI, Antonio. Producción de pobreza y desigualdad en América Latina. Buenos Aires: CLACSO/ Del Hombre, 2008. p. 41-77.

NELSON, Ernesto ¿Por qué delinquen nuestros niños? Revista de Criminología, Psiquiatría y Medicina Legal, Buenos Aires, v. 2, p. 830-860, 1935.

NELSON, Ernesto. Hacia un programa coordinado de prevención de la delincuencia infantil. Revista de Criminología, Psiquiatría y Medicina Legal, Buenos Aires, v. 12, p. 220-234, 1945.

PACHÓN, Ximena. La casa de corrección de Paiba en Bogotá. En: RODRíGUEZ, Pablo; MANNARELLI, María Emma (Comp.). Historia de la Infancia en América Latina. Bogotá: Universidad Externado de Colombia, 2007. p. 323-339.

PONCE, Luis Adolfo. Los Tribunales de Menores en la Provincia de Buenos Aires. Revista de Criminología, Psiquiatría y Medicina Legal, v. 3, p. 306-314, 1950.

QUEIROLO, Graciela. Malos pasos y promociones: aproximaciones al trabajo femenino asalariado desde la historia y la literatura (Buenos Aires, 1919-1939). Anuario de la Escuela de Historia, Rosario, v. 22, p. 53-80, 2011. Disponible en: http://hdl.handle.net/2133/3661. Acceso en: 2 sept. 2021.

RECA, Telma. Estudio social del niño delincuente, Revista de Criminología, Psiquiatría y Medicina Legal, Buenos Aires, v. 2, p. 157-163, 1932.

RAMACCIOTTI, Karina Inés. Telma Reca en la gestión estatal de la sanidad argentina (19301948). Asclepio, Madrid, v. 70. n. 1, pág. 2-13. 2018. https://doi.org/10.3989/asclepio.2018.04

RIBEIRO, Leónidio. Revelación de anomalías en los niños desde el punto de vista de la prevención criminal. Revista de Criminología, Psiquiatría y Medicina Legal, Buenos Aires, v. 3 , p. 445-447, 1936. 
RODRÍGUEZ LÓPEZ, Carmen. La niñez abandonada y delincuente en las dos primeras décadas del siglo XX: camino a la ley agote. Revista de Historia del Derecho, Buenos Aires, v. 44, p. 177-200, 2012.

ROSSI, Arturo; GRAFIGNA, Ángel. Biotipología como fundamento de la reforma escolar. Anales de Biotipología, Eugenesia y Medicina Social, Buenos Aires, v. 5, p.11-12,1938.

ROSSI, Arturo. Antropología, Criminología y Biotipología criminal. Anales de la Sociedad Argentina de Criminología, Buenos Aires, v. 9, p. 173-234, 1943.

ROXO, Enrique. Educación y tratamiento de niños anormales. Revista de Criminología, Psiquiatría y Medicina Legal, Buenos Aires, v. 3, p. 494-504, 1936.

SOSENSKY, Susana. Un remedio contra la delincuencia: el trabajo infantil en las instituciones de encierro de la Ciudad de México durante la posrevolución. Asclepio, Madrid, v. 60. n. 2, p. 95-118, 2008. https://doi.org/10.3989/asclepio.2008.v60.i2.259

SMYTHE, Julio Altman. El problema del menor en estado peligroso. Revista de Criminología, Psiquiatría y Medicina Legal, Buenos Aires, v. 2, p. 408-420, 1934.

STAGNO, Leandro. La configuración de la juventud como un problema: delitos y vida cotidiana de varones jóvenes provenientes de los sectores populares (La Plata, 1938-1942). 2019. Tesis (Doctorado en Ciencias sociales)-Facultad Latinoamericana de Ciencias Sociales, Buenos Aires, 2020. Disponible en: https://www.memoria.fahce.unlp.edu.ar/tesis/te.1868/te.1868.pdf. Acceso en: 22 agosto 2021.

TALAK, Ana. Eugenesia e higiene mental: usos de la psicología en la Argentina, 1900-1940. En: MIRANDA, Marisa; VALLEJO, Gustavo (Comp.). Darwinismo social y eugenesia en el mundo latino. Buenos Aires: Siglo XXI, 2005. p. 563-599.

VALLEJO, Gustavo; MIRANDA, Marisa. Los saberes del poder: eugenesia y biotipología en Argentina del siglo XX. Revista de Indias, Madrid, v.64, n. 231, p. 425-444, 2004. Disponible en: https://core.ac.uk/download/pdf/267885474.pdf. Acceso en: 22 agosto 2021.

ZAPIOLA, María Carolina. Aproximaciones científicas a la cuestión del delito infantil: el discurso positivista en los Archivos de Psiquiatría, Criminología y Ciencias Afines, Argentina, comienzos del siglo XX. En: SOZZO, Máximo (Coord.). Historia de la cuestión criminal en la Argentina: Buenos Aires (Argentina). Buenos Aires: Del Puerto, 2009. p. 50-74. 